\title{
Validação da Escala de Abordagens de Aprendizagem (EABAP) em uma Amostra Brasileira
}

\author{
Validation of the Learning Approach Scale (LAS) in a Brazilian Sample
}

\author{
Cristiano Mauro Assis Gomes*, Hudson Fernandes Golino, Carlos Alberto Ribeiro Pinheiro, \\ Gregório Ribeiro Miranda, \& Juliana Marina Tavares Soares \\ Universidade Federal de Minas Gerais, Belo Horizonte, Brasil
}

\begin{abstract}
Resumo
O presente estudo teve como objetivo validar a Escala de Abordagens de Aprendizagem (EABAP), construída para mensurar a dimensão Superficial e Profunda, em uma amostra brasileira. Fizeram parte da pesquisa 716 estudantes do ensino fundamental e médio de uma escola de Belo Horizonte. A análise fatorial exploratória foi utilizada para a identificação das dimensões e seleção dos melhores itens. Dos 27 itens estipulados, foram mantidos 17. A Análise Fatorial Confirmatória indicou a adequação da solução de um fator geral e de dois fatores específicos que representam a Abordagem Superficial e a Profunda $(\chi 2=281,50$; $\mathrm{gl}=116 ; C F I=0,94 ; G F I=0,95$ e RMSEA=0,05). São discutidas implicações dos resultados para a teoria das abordagens, assim como algumas potencialidades do EABAP para a Psicologia Educacional.

Palavras-chave: Abordagem profunda; Aprendizagem; Validação.
\end{abstract}

\begin{abstract}
The main objective of the current study is to build and validate the Learning Approach Scale (LAS), which was developed to assess the deep and surface dimensions in a Brazilian sample. Seven hundred sixteen participants from junior and high schools in the city of Belo Horizonte took part in the research. The exploratory factor analysis was used to identify those dimensions and to select the best items. Only 17 items were kept from the 27 original ones. By the Confirmatory Factor Analysis it was possible to identify the adequacy of one general and two specific factors, the latter representing the deep and surface approaches $(\chi 2=281.50 ; \mathrm{gl}=116 ; C F I=0.94 ; G F I=0.95$ and $\mathrm{RMSEA}=0.05)$. The implications of the results for the theory of approaches and the application of the scale in the Educational Psychology field are discussed.

Keywords: Deep approach; Learning process; Factorial validation.
\end{abstract}

\section{Abordagens de Aprendizagem}

Segundo Boruchovitch (1999), estudos evidenciam que as escolas, em sua maioria, não têm intervindo adequadamente no processo de "aprender a aprender" e "aprender a pensar" dos estudantes. Ao contrário, sustentam sua proposta pedagógica no modelo de transmissão da informação. Paradoxalmente, elas demandam habilidades cognitivas de alto nível, como análise, dedução, inferência lógica, interpretação de elementos implícitos, entre outras, no cotidiano dos alunos (Almeida, 1996; Boruchovitch, 1999; Mettrau \& Mathias, 1998). Essa condição faz com que pesquisadores salientem o desajuste entre o que a escola exige e proporciona (Almei-

\footnotetext{
*Endereço para correspondência: Universidade Federal de Minas Gerais, Faculdade de Filosofia e Ciências Humanas, Departamento de Psicologia, Av. Antônio Carlos, 6627, Sala 4010, Pampulha, Belo Horizonte, MG, Brasil, CEP 31270-901. E-mails: cristianogomes@ufmg.br ou cgomes@fafich.ufmg.br

Apoio Fundação de Amparo à Pesquisa do estado de Minas Gerais (FAPEMIG).
}

da, 1992; Gomes, 2005). Tanto na educação básica quanto na educação superior, há ocorrência de déficits de desempenho e problemas de compreensão do conteúdo estudado (Almeida, 1996; Duarte, 2002; Gomes, 2005). Esses estudos apontam, também, a necessidade de se investigar os processos envolvidos na aprendizagem e no desempenho escolar, e demonstram a insuficiência do modelo transmissivo na educação.

A aprendizagem, pensada em termos da compreensão do conteúdo pelo aluno e seu desempenho acadêmico, é influenciada por uma diversidade de fatores: a capacidade metacognitiva e de processamento das informações, a motivação, o contexto de ensino-aprendizagem, o ambiente familiar e social dos alunos, os objetivos e métodos de ensino, entre outros (Biggs, 1987a; Boruchovitch, 1999; Duarte, 2002; Gomes, 2005; Hattie, Biggs, \& Purdue, 1996; Holt, 1982; Struyven, Dochy, Janssens, \& Gielen, 2006; Wild \& Quinn, 1998). Entre esses fatores, as estratégias de aprendizagem influenciam o aprendizado e o desempenho dos estudantes, na medida em que se constituem como um conjunto de técnicas, ferramentas e 
seqüências de procedimentos ou atividades utilizadas pelos indivíduos para adquirir, armazenar e utilizar a informação (Dembo, 1994; Nisbet \& Schucksmith, 1986; Pozo, 1996; Riding \& Rayner, 1998). Há evidências de que a utilização de certas estratégias de aprendizagem influencia positivamente o desempenho acadêmico, contribui para uma aprendizagem efetiva, possibilita a superação de dificuldades pessoais e ambientais, e potencializa a aprendizagem (Da Silva \& Sá, 1993; Garner, Hare, Alexander, Haynes, \& Winograd, 1984; Pressley \& Levin, 1983; Weinstein \& Mayer, 1985). O estudo dessas estratégias contribui para uma maior compreensão acerca do desempenho dos estudantes, pois auxilia na identificação e prevenção de problemas de aprendizagem em idade precoce e fornece conhecimentos que podem ajudar a promover intervenções educacionais capazes de ampliar a capacidade de aprender dos alunos (Boruchovitch, 1993).

Há uma tradição de investigação no campo das estratégias denominadas abordagens de aprendizagem (Biggs, 1987a; Entwistle, 1988; Marton, 1975). Influenciada por teorias construtivistas e do processamento da informação, essa tradição valoriza processos de ensino e aprendizagem que envolvem a participação ativa do estudante na seleção, interpretação e aplicação do conhecimento, possibilitando um aprendizado mais profundo. Distancia-se, assim, dos modelos tradicionais, transmissivos, focados na reprodução das informações, nos quais o aluno é passivo no processo de aquisição do conhecimento (Boruchovitch, 1999; De Corte, 2000; Struyven et al., 2006)

O campo de estudo das abordagens de aprendizagem surgiu a partir dos estudos seminais de Marton e Saljö (1976a, 1976b). Eles detectaram diferenças nos comportamentos dos estudantes ao lidar com uma tarefa de leitura e compreensão de textos, delimitando duas abordagens, Profunda e Superficial. A partir desses estudos, outros pesquisadores buscaram identificar empiricamente estes construtos (Entwistle, McCune, \& Walker, 2001; Entwistle, Meyer, \& Tait, 1991; Entwistle \& Ramsden, 1983; Struyven et al., 2006; Vermunt \& Vermetten, 2004). Em suas pesquisas, Biggs $(1985,1987 a)$ identificou uma terceira Abordagem, denominada Estratégica.

Os pesquisadores dessa tradição têm preferido o termo Abordagem ao termo Estratégia na medida em que, tanto do ponto de vista teórico quanto empírico, as estratégias de aprendizagem não se encontram dissociadas da motivação para aprender. A definição de Abordagem representa a relação imbricada e indissolúvel entre motivação para aprender e estratégias de aprendizagem.

A Abordagem Profunda $(A P)$ envolve, cognitiva e afetivamente, uma postura ativa do sujeito no processo de aprendizagem. São características dessa Abordagem a interpretação de aspectos explícitos e implícitos, a construção de relações amplas e abstratas, assim como a busca pela compreensão e formação de significados pessoais frente aos objetos de conhecimento. Nessa abordagem o componente afetivo envolve o interesse intrínseco no que se aprende e no desenvolvimento de competências acadêmicas.

A Abordagem Superficial $(A S)$, por sua vez, conduz a uma retenção de detalhes fatuais, através de memorização para a reprodução dos mesmos, o que dificulta a identificação de um conjunto mais amplo a respeito das relações existentes e limita a interação entre o sujeito e os objetos de conhecimento. Nessa abordagem, o componente afetivo é direcionado a uma motivação extrínseca à tarefa (Biggs, 1987a; Entwistle \& Ramsden, 1983; Riding \& Rayner, 1998; Struyven et al., 2006; Vermunt \& Vermetten, 2004; Wild \& Quinn, 1998).

Enquanto a $A P$ e $A S$ definem duas maneiras do estudante lidar com o conhecimento, a Abordagem Estratégica $(A E)$ se caracteriza pela capacidade do aluno em transitar entre essas duas abordagens, visando obter uma boa nota escolar (Biggs, 1987a). O aluno funciona a partir da $A S$ quando a atividade escolar requer apenas memorização e reprodução da informação. Quando a atividade demanda formação de relações, interpretação de elementos implícitos, dentre outros fatores, o aluno funciona a partir da $A P$. Segundo Biggs (1987a), a $A E$ ocorre em função da regulação da conduta voltada a atingir a maximização do desempenho escolar. Isso indica que muito provavelmente o tipo de abordagem depende fortemente do tipo de tarefa exigida na escola.

$\mathrm{Na}$ tentativa de identificação empírica das abordagens de aprendizado, várias baterias foram elaboradas. Dentre elas pode-se citar o Learning Process Questionnaire (Biggs, 1985, 1987a, 1987b), o Study Process Questionnaire (Biggs, 1978, 1987b), e suas versões reformuladas, o R-SPQ-2F (The Revised Two - Factor Study Process Questionnaire) e o RLPQ-2F (The Revised Two - Factor Learning Process Questionnaire), de Biggs e colaboradores (Biggs, Kember, \& Leung, 2001). Podese também pontuar, entre outras baterias, o Approaches to Study Inventory, de Entwistle e Ramsden (1983), a Escala de Avaliação de Processos de Estudo (Gomes, 2005), e a versão em português do Study Process Questionnaire, denominada Questionário de Processos de Aprendizagem, desenvolvida por Rosário e colaboradores (Rosário, Almeida, Núñez, \& González-Pienda, 2004).

\section{Escala de Abordagens de Aprendizagem (EABAP)}

A Escala de Abordagens de Aprendizagem foi elaborada no ano de 2007 pelo primeiro e pelo último autor deste artigo. Os itens da escala foram construídos levando-se em consideração a $A S$ e $A P$, exclusivamente. Esta opção se deve a dois motivos. O primeiro remete à necessidade de evidências mais efetivas para a comprovação da existência da $A E$. O segundo remete ao fato de que as versões atuais dos instrumentos de Biggs não incluem mais a $A E$ em seu universo de mensuração, medindo exclusivamente as duas abordagens clássicas, superficial 
e profunda. Wong, Lin e Watkins (1996) realizaram um estudo transcultural confirmatório com amostras de diversos países e verificaram que apenas o modelo das abordagens superficial e profunda pode ser considerado robusto.

A EABAP é uma escala de auto-relato formada por 27 itens. Cada item possui um enunciado que remete a um comportamento do estudante frente à aprendizagem. $\mathrm{O}$ aluno julga o quanto do comportamento descrito está presente em sua vida, utilizando uma escala do tipo Likert que varia de (1) nem um pouco até (5) totalmente.

Os itens da EABAP, em geral, articulam uma relação intrínseca entre motivação e estratégia. Os enunciados, majoritariamente, designam tanto estratégias quanto motivações para aprender. $\mathrm{O}$ item "Eu só estudo na véspera das provas, decorando a matéria" exemplifica essa relação. A conduta descrita no item, muito provavelmente, indica um baixo engajamento do aluno em relação à construção do conhecimento, e uma ausência de motivação intrínseca frente à aprendizagem escolar. Concomitante, esta conduta indica uma estratégia de estudo e aprendizagem superficial. Apesar da relação intrínseca entre motivação e estratégias, os itens apresentam um foco específico no aspecto motivacional ou estratégico. Nesse exemplo, há um foco no componente estratégico, uma vez que esse tipo de item enfatiza maneiras de fazer e escolhas de ação. Por outro lado, os itens que enfatizam preferências, gostos, e aspectos afetivos, possuem foco em componentes motivacionais. $\mathrm{O}$ item "Eu gosto de atividades que exigem uma reflexão sobre o assunto para sua execução" exemplifica o foco na dimensão motivacional. Em função do foco motivacional ou estratégico, podem-se classificar os itens como: motivação superficial, motivação profunda; estratégia superficial, estratégia profunda.

Apesar de apresentar consistência teórica e metodológica, a EABAP necessita de investigações que avaliem sua validade e confiabilidade. O presente artigo apresenta o primeiro relato da validade da EABAP, em nível exploratório, e discute algumas implicações e contribuições ligadas à identificação das abordagens.

\section{Método}

\section{Participantes}

Fizeram parte da amostra 716 alunos de uma instituição particular de ensino fundamental e médio de Belo Horizonte, Minas Gerais, sendo 379 (52,93\%) do gênero feminino, com idade variando entre 8 e 18 anos ( $m=13,75$ e $D P=2,11)$. O número de participantes por série foi equilibrado. A sexta série apresentou o menor número de alunos (94), com $13,13 \%$ do total da amostra, e a nona série o maior número de alunos (119), com $16,62 \%$ da amostra. Do total de participantes, 58,94\% (422 alunos) eram oriundos do ensino fundamental.

\section{Instrumento}

Escala de Abordagens de Aprendizagem. A EABAP é um instrumento de auto-relato composto por 27 itens respondidos de acordo com uma escala do tipo Likert contendo (1) Nem um pouco, (2) Um pouco, (3) Mais ou menos, (4) Muito e (5) Totalmente. Essa escala se refere ao quanto do comportamento descrito no item o aluno acredita corresponder à sua maneira de aprender. Ela pode ser aplicada individual ou coletivamente e não possui limite de tempo, apesar de não demandar mais de 15 minutos para sua realização.

\section{Coleta e Análise de Dados}

A aplicação do instrumento foi realizada no início de 2008, em 19 turmas de uma escola particular de Belo Horizonte, sendo treze do ensino fundamental e seis do ensino médio. A EABAP foi aplicada de forma coletiva, e sempre por psicólogos ou estudantes de psicologia devidamente treinados. Foram tomados todos os cuidados éticos em relação aos participantes e a pesquisa contou com a aprovação do Comitê de Ética da Universidade Federal de Minas Gerais (UFMG) - n. ETIC 456/07.

Para a identificação das abordagens de aprendizagem foram utilizados os procedimentos de análise fatorial exploratória e confirmatória. A análise fatorial exploratória foi usada inicialmente, através do procedimento dos eixos principais, para a primeira identificação de soluções possíveis e eliminação de itens e fatores mal identificados. Para a análise da retenção dos fatores, foram utilizados os seguintes procedimentos fatoriais exploratórios: auto-valor-maior-do-que-um, scree test, análise paralela por permutação e máxima verossimilhança. Esses procedimentos foram selecionados, uma vez que eles tendem a gerar diferentes soluções (Beauducel, 2001; Frazier \& Youngstrom, 2007; Guadagnoli \& Velicer, 1988; Henson \& Roberts, 2006). Para as soluções identificadas, foi usada a rotação oblíqua promax quatro.

Alguns critérios foram combinados ou utilizados isoladamente para a eliminação de algum item do instrumento. Se um item possuísse carga fatorial inferior a 0,30 em relação ao seu fator de referência no primeiro nível, ele poderia ser eliminado, assim como se possuísse carga fatorial superior a $0,30 \mathrm{em}$ mais de um fator. Um item também poderia ser eliminado caso ele se relacionasse diretamente a um fator mal definido, com poucos itens ou com um alpha de Cronbach muito baixo (menor do que 0,50 ). Ao eliminar os itens, os pesquisadores não sabiam o seu conteúdo, observando exclusivamente o valor psicométrico destes. Essa estratégia buscou eliminar o viés de escolha e distribuição dos itens de acordo com a abordagem.

A análise fatorial confirmatória foi empregada para verificar se a solução exploratória final apresentava bom ajuste aos dados. O grau de ajuste foi avaliado através dos índices Comparative Fit Index (CFI), Goodness-offit index (GFI) e o Root Mean Square Error Approximation 
(RMSEA). Os dois primeiros precisam ter valor igual ou superior a 0,90 , enquanto o $R M S E A$ deve possuir uma carga igual ou inferior a 0,08 para o grau de ajuste do modelo ser considerado adequado (Blunch, 2008; Byrne, 2001).

\section{Resultados}

Uma série de modelos exploratórios foi gerada a partir da eliminação de itens e fatores mal identificados. No final desse processo identificou-se um modelo considerado consistente e satisfatório, pois apresentava fatores com um alpha igual ou superior a 0,70 , e itens com carga igual ou superior a $0,30 \mathrm{em}$ apenas um fator de primeiro nível.

A solução exploratória final apresentou dois fatores de primeira ordem e uma correlação entre eles $(r=-0,44)$, caracterizando uma evidência favorável a respeito da presença de um fator geral de segunda ordem. Essa solução final foi desenhada em um modelo com uma estrutura fatorial hierárquica de dois níveis, sendo um fator geral de segunda ordem e dois fatores específicos de primeira ordem. A análise fatorial confirmatória indicou a adequação do modelo $\left(\chi^{2}=281,50 ; \mathrm{gl}=116 ; C F I=0,94 ; G F I\right.$ $=0,95$ e RMSEA $=0,05)$.

Dos 27 itens iniciais, 10 foram eliminados. Dos 17 itens restantes, todos são carregados pelo fator geral, nove são carregados pelo fator 1 e oito pelo fator 2 (Tabela 1 ).
Nenhum possui carga inferior a 0,43 em relação ao seu fator de referência, o que os aponta como bons marcadores. A distribuição de itens por fatores permite dizer que o fator 1 representa uma abordagem profunda e o fator 2 uma abordagem superficial. Selecionando, como exemplo, os dois itens com melhores cargas em cada fator (sendo 8 e 26 do fator 1; 16 e 20 do fator 2) tem-se:

8. Eu busco me envolver em atividades que aumentem o meu conhecimento.

26. Eu vejo nos estudos uma possibilidade de desenvolver meu pensamento.

16. Eu só leio os textos que o professor manda.

20. Eu só estudo quando sou obrigado.

Os itens 8 e 26 demonstram engajamento na busca pelo conhecimento, atitude ativa do aluno frente ao seu processo de aprendizagem e a relação intrínseca entre estratégia e motivação. Já os itens 16 e 20 indicam atitude passiva do aluno, dependência em relação ao professor e falta de motivação intrínseca pelo estudo. Dos itens da $A P$, cinco $(55,55 \%)$ descrevem aspectos motivacionais $\mathrm{e}$ quatro $(44,45 \%)$ descrevem estratégias de aprendizagem. Esse equilíbrio é, também, encontrado nos itens da $A S$ que apresenta três itens relacionados aos aspectos motivacionais e cinco itens relacionados às estratégias.

$\mathrm{O}$ fator geral foi carregado adequadamente por todos os itens, com cargas variando entre 0,29 e 0,43 . Os itens do fator de $A P$ apresentaram uma polaridade positiva em relação ao fator geral, enquanto os itens da $A S$ apresenta-

Tabela 1

Análise Fatorial Confirmatória - População, Ensino Fundamental e Médio

\begin{tabular}{lccccccccc}
\hline & ag & ag1 & ag2 & as & as1 & as2 & ap & ap1 & ap2 \\
\hline as & 0,68 & 0,62 & 0,74 & 0,00 & 0,00 & 0,00 & 0,00 & 0,00 & 0,00 \\
ap & 0,68 & 0,62 & 0,74 & 0,00 & 0,00 & 0,00 & 0,00 & 0,00 & 0,00 \\
i02 & 0,37 & 0,31 & 0,44 & 0,55 & 0,50 & 0,60 & 0,00 & 0,00 & 0,00 \\
i07 & 0,38 & 0,34 & 0,40 & 0,56 & 0,56 & 0,53 & 0,00 & 0,00 & 0,00 \\
i09 & 0,32 & 0,24 & 0,45 & 0,47 & 0,39 & 0,60 & 0,00 & 0,00 & 0,00 \\
i12 & 0,30 & 0,23 & 0,41 & 0,45 & 0,38 & 0,55 & 0,00 & 0,00 & 0,00 \\
i14 & 0,29 & 0,22 & 0,38 & 0,43 & 0,36 & 0,51 & 0,00 & 0,00 & 0,00 \\
i16 & 0,41 & 0,34 & 0,49 & 0,61 & 0,55 & 0,66 & 0,00 & 0,00 & 0,00 \\
i19 & 0,38 & 0,31 & 0,45 & 0,56 & 0,51 & 0,61 & 0,00 & 0,00 & 0,00 \\
i20 & 0,39 & 0,34 & 0,46 & 0,57 & 0,54 & 0,62 & 0,00 & 0,00 & 0,00 \\
i08 & 0,43 & 0,40 & 0,47 & 0,00 & 0,00 & 0,00 & 0,64 & 0,64 & 0,64 \\
i10 & 0,38 & 0,38 & 0,37 & 0,00 & 0,00 & 0,00 & 0,55 & 0,59 & 0,50 \\
i13 & 0,41 & 0,39 & 0,45 & 0,00 & 0,00 & 0,00 & 0,61 & 0,62 & 0,60 \\
i15 & 0,41 & 0,40 & 0,41 & 0,00 & 0,00 & 0,00 & 0,60 & 0,63 & 0,56 \\
i22 & 0,38 & 0,32 & 0,47 & 0,00 & 0,00 & 0,00 & 0,56 & 0,51 & 0,63 \\
i23 & 0,40 & 0,31 & 0,53 & 0,00 & 0,00 & 0,00 & 0,59 & 0,50 & 0,72 \\
i25 & 0,40 & 0,34 & 0,47 & 0,00 & 0,00 & 0,00 & 0,59 & 0,55 & 0,64 \\
i26 & 0,42 & 0,43 & 0,40 & 0,00 & 0,00 & 0,00 & 0,62 & 0,70 & 0,54 \\
i27 & 0,40 & 0,35 & 0,47 & 0,00 & 0,00 & 0,00 & 0,59 & 0,57 & 0,63 \\
alpha & 0,84 & 0,84 & 0,94 & 0,75 & 0,69 & 0,81 & 0,84 & 0,84 & 0,85
\end{tabular}

Nota. $a g$ = fator geral da população; $a g 1$ = fator geral do ensino fundamental; $a g 2$ = fator geral do ensino médio; $a s=$ abordagem superficial da população; as $1=$ abordagem superficial do ensino fundamental; as 2 = abordagem superficial do ensino médio; $a p=$ abordagem profunda da população; apl = abordagem profunda do ensino fundamental; ap 2 = abordagem profunda do ensino médio. 
ram uma polaridade negativa. Essa condição demonstra que cada um dos extremos da escala indica uma das abordagens. Dessa forma, uma alta pontuação no fator geral indica uma intensa $A P$, e uma baixa pontuação aponta a presença de uma forte $A S$.

O modelo composto por um fator geral e dois fatores específicos possui ajuste adequado tanto para o ensino fundamental quanto para o ensino médio. Os índices estatísticos do modelo referente ao ensino médio foram $\chi^{2}$ $=191,30, \mathrm{gl}=116, C F I=0,95, \mathrm{GFI}=0,93$ e RMSEA $=$ 0,05 . Para o ensino fundamental os índices estatísticos foram $\chi^{2}=237,60, \mathrm{gl}=116, C F I=0,92, \mathrm{GFI}=0,94 \mathrm{e}$ RMSEA $=0,05$.

Através da ANOVA, constatam-se diferenças no desempenho médio dos alunos com relação à série, tanto na $A P(F=4,25 ; \mathrm{gl}=6 ; p=0,00)$ quanto na $A S(F=3,22$; $\mathrm{gl}=6 ; p=0,00)$. Há uma diminuição das médias da $A P$ entre a sexta e a nona série, que se recuperam, progressivamente, ao longo ensino médio. No entanto, as médias de desempenho em $A S$ mantém-se relativamente estáveis até a nona série do ensino fundamental, que por sua vez diminuem ao longo do ensino médio (Tabela 2 e Figura 1). Essa constatação acentua o ganho médio da $A P$ no ensino médio.

Tabela 2

Média (m) e Desvio-Padrão (DP) nas Abordagens por Série

\begin{tabular}{ccccc}
\hline & \multicolumn{2}{c}{ profunda } & \multicolumn{2}{c}{ superficial } \\
\cline { 2 - 5 } & $\mathrm{m}$ & DP & $\mathrm{m}$ & DP \\
\hline 06 & 0,31 & 0,97 & 0,06 & 0,88 \\
07 & 0,07 & 1,02 & 0,11 & 0,91 \\
08 & $-0,09$ & 0,86 & 0,08 & 0,78 \\
09 & $-0,25$ & 0,80 & 0,10 & 0,81 \\
10 & $-0,13$ & 0,95 & 0,01 & 0,92 \\
11 & 0,00 & 0,86 & $-0,08$ & 0,85 \\
12 & 0,14 & 0,91 & $-0,32$ & 0,96 \\
\hline
\end{tabular}

Nota. $06=6^{\mathrm{a}}$ série; $07=7^{\mathrm{a}}$ série; $08=8^{\mathrm{a}}$ série; $09=9^{\mathrm{a}}$ série; 10 $=1^{\mathrm{a}}$ série do ensino médio; $11=2^{\mathrm{a}}$ série do ensino médio; $12=$ $3^{\mathrm{a}}$ série do ensino médio.

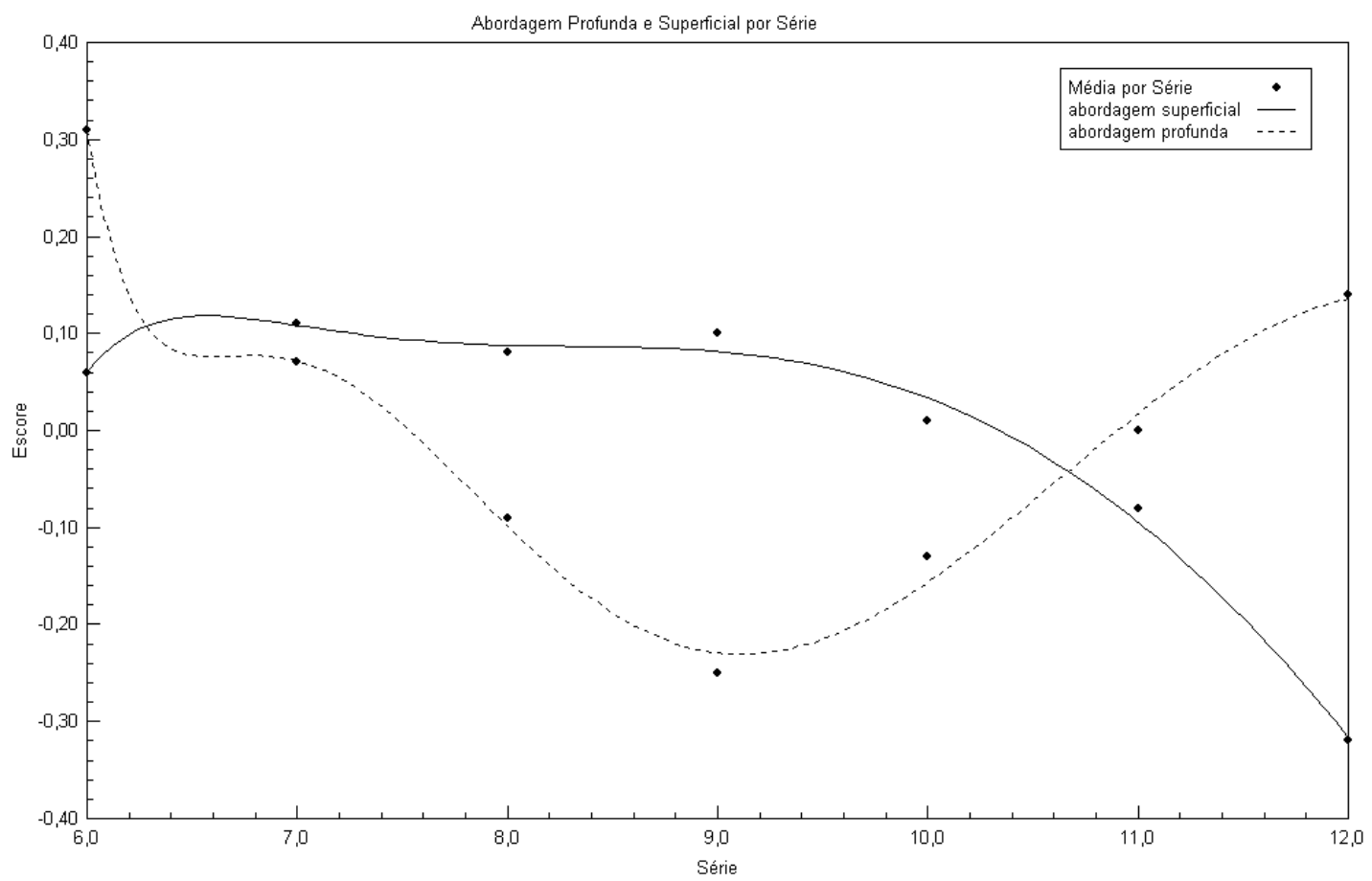

Figura 1. Média de desempenho nas abordagens por série

Há, também, diferenças em relação às médias de desempenho de acordo com a idade, tanto para $A P(F=$ $3,42 ; \mathrm{gl}=9 ; p=0,00)$ quanto para $A S(F=2,13 ; \mathrm{gl}=9$; $p=0,03)$. Há uma relativa estabilidade na média em $A S$ entre os participantes de 10 aos 14 anos e uma tendência de diminuição das médias a partir dos 15 anos que, no entanto, volta a aumentar entre os participantes com 18 anos. Porém, deve-se considerar o pequeno número de casos $(n=9)$ nesta faixa etária. Já a média de desempe- nho em $A P$ não apresenta um quadro de estabilidade em nenhum intervalo de idade. Há uma queda nas médias entre os participantes de 10 aos 14, e um aumento a partir dos 15 anos (Tabela 3 e Figura 2). Os participantes com oito anos não foram considerados na análise etária devido ao baixo número $(n=2)$. A média e desvio padrão em cada uma das abordagens foram especificados a partir dos dez anos (Tabela 3). 
Tabela 3

Média (m) e Desvio-Padrão (DP) nas Abordagens por Idade

\begin{tabular}{lrrrr}
\hline & \multicolumn{2}{c}{ profunda } & \multicolumn{2}{c}{ superficial } \\
\cline { 2 - 5 } idade & $\mathrm{m}$ & $\mathrm{DP}$ & $\mathrm{m}$ & $\mathrm{DP}$ \\
\hline 10 & 0,38 & 1,01 & 0,08 & 0,85 \\
11 & 0,29 & 0,92 & 0,00 & 0,90 \\
12 & 0,01 & 0,98 & 0,14 & 0,83 \\
13 & $-0,16$ & 0,83 & $-0,05$ & 0,72 \\
14 & $-0,19$ & 0,87 & 0,13 & 0,87 \\
15 & 0,01 & 0,90 & $-0,06$ & 0,90 \\
16 & $-0,09$ & 0,97 & $-0,1$ & 0,99 \\
17 & 0,07 & 0,88 & $-0,21$ & 0,93 \\
18 & $-0,11$ & 0,66 & 0,22 & 0,71 \\
\hline
\end{tabular}

Diferentemente das variáveis série e idade, gênero apresenta diferenças significativas na média dos desempenhos apenas na abordagem superficial $(F=37,80 ; \mathrm{gl}=1 ; p=$ $0,00)$. Estudantes do sexo masculino possuem médias mais altas em $A S(m=0,21$ e $D P=0,84)$ do que estudantes do sexo feminino ( $m=-0,19$ e $D P=0,87)$.

\section{Discussão}

Os resultados deste estudo mostram que é possível identificar as duas abordagens clássicas, $A P$ e $A S$, em amostras brasileiras. A distribuição dos itens nos fatores de primeiro nível identificados corrobora a relação imbricada entre estratégia e motivação, definidora do conceito de abordagem. Todos os fatores identificados foram carre-

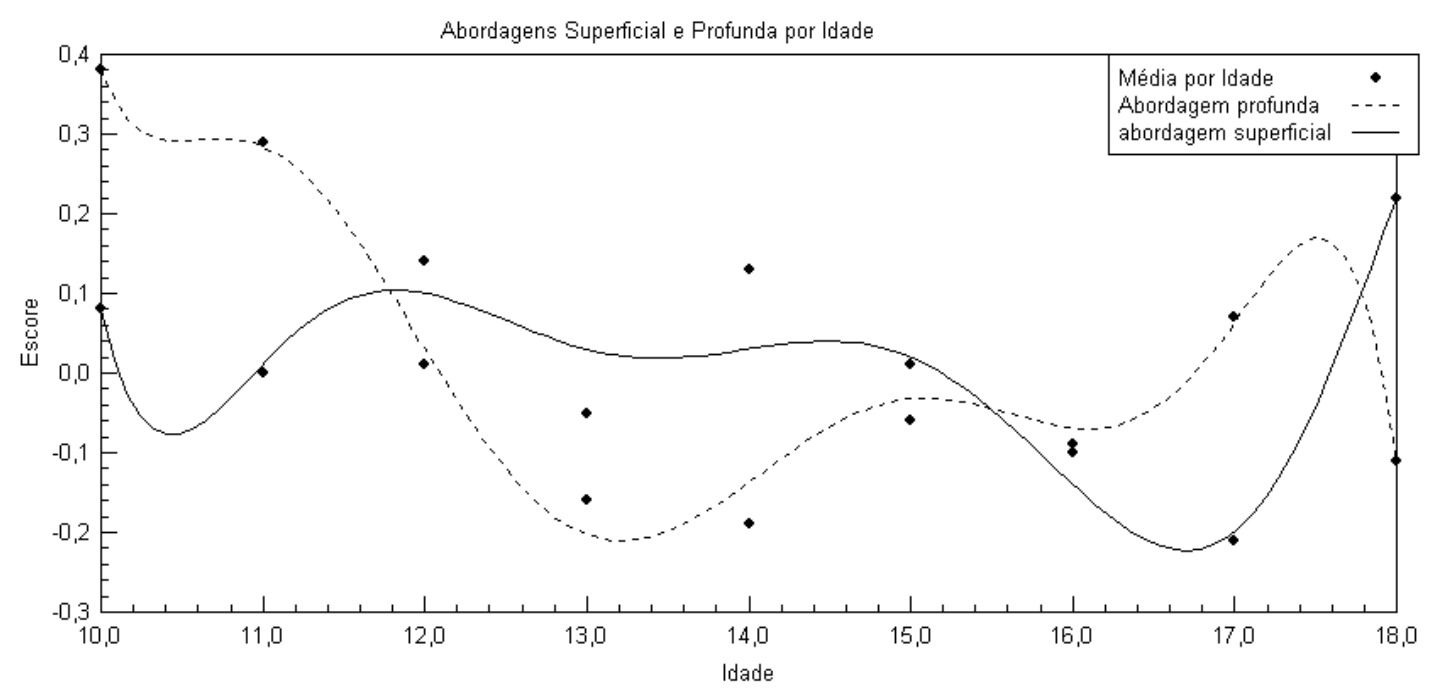

Figura 2. Média de Desempenho nas Abordagens por Idade

gados por uma mescla equilibrada de itens envolvidos nesses dois domínios. Buscando evitar que essa articulação fosse devido a um artefato metodológico, a eliminação dos itens do instrumento foi feita através de análise cega. Os pesquisadores não tinham acesso ao conteúdo do item que eliminavam, de forma que não sabiam se retiravam um item com foco em motivação ou em estratégia, ou relacionado à abordagem superficial ou à abordagem profunda.

Quanto à escala obtida, pode-se argumentar que um escore geral é adequado para mensurar as duas abordagens, na medida em que o fator geral identificado apresenta uma polaridade positiva frente à abordagem profunda e uma polaridade negativa em relação à abordagem superficial. Essa condição implica em uma medida na qual cada um dos extremos caracteriza uma das abordagens. Quanto maior a pontuação na escala, maior a abordagem profunda e menor a superficial. Por outro lado, a utilização de uma escala separada para cada abordagem é possível, na medida em que este estudo identificou um fator específico, bem mensurado e confiável para cada uma das abordagens. Nesse sentido, pode-se tanto utilizar um escore geral quanto escores específicos, dependendo do foco de análise e do objetivo da investigação. Nos resultados desse artigo, foram utilizados os escores específicos para que se pudesse dispor de uma informação mais ampla sobre possíveis trajetórias nas diferentes abordagens.

Analisando as implicações do escore geral, onde os extremos identificam os tipos de abordagem, pode-se perguntar qual o ponto desses extremos é capaz de situar, de maneira sensível e específica, se um determinado desempenho indica claramente uma abordagem profunda ou superficial. Para responder a essa questão, são necessários estudos posteriores capazes de definir e empregar critérios diagnósticos das duas abordagens e utilizar o método da curva ROC para identificar qual ponto da escala possui adequada sensibilidade e especificidade.

Apesar das possibilidades advindas dos novos estudos sugeridos, uma questão permanece em aberto e se refere ao desempenho situado em pontos medianos da escala. $\mathrm{O}$ que significa o desempenho localizado em pontos próxi- 
mos à média da escala geral? Sabe-se, apenas, que eles não atendem aos critérios mais rigorosos de diagnóstico focado na especificidade. Na medida em que eles não apontam uma forte abordagem profunda ou superficial, pode-se especular uma possível relação com a abordagem estratégica proposta por Biggs (1987a). No entanto, é preciso investigar empiricamente esta especulação através de estudos capazes de verificar se há uma maior facilidade pelo emprego funcional da abordagem estratégica pelas pessoas com desempenho próximo à média, ou seja, pessoas que não apresentam uma forte abordagem profunda ou superficial. Por outro lado, esses estudos deverão considerar o aspecto motivacional, uma vez que Biggs (1987a) explica que a abordagem estratégica depende do foco na obtenção de um alto desempenho acadêmico.

As diferenças em $A P$ e $A S$, verificadas através do desempenho médio por série, idade e gênero, retratam dados transversais que não possibilitam conclusões acerca das trajetórias de desenvolvimento. Por outro lado, dados transversais permitem a elaboração de hipóteses e especulações sobre possíveis caminhos de desenvolvimento. Um dado que merece ser investigado futuramente diz respeito à curva em formato de "U" do desempenho médio em $A P$, de acordo com as séries. Esse dado, caso seja encontrado em estudos longitudinais, pode ser um retrato localizado e específico de uma cena mais abrangente, uma vez que esse formato de curva de desempenho pode indicar possíveis trajetórias descontínuas, envolvendo uma desestabilização e queda funcional devido à aquisição de uma nova habilidade (Fischer \& Kennedy, 1997). Dawson-Tunik, Commons, Wilson e Fischer (2005), Fischer e Bidell (2006), assim como Yan e Fischer (2002) mostram que curvas do tipo $U$ ou do tipo $\mathrm{V}$ demonstram, mesmo em dados de recorte transversal, uma variabilidade típica do desenvolvimento de novas habilidades cognitivas. É possível que a queda verificada em $A P$ se relacione com o desenvolvimento de um novo estágio cognitivo, operatório formal.

Do ponto de vista educacional, a teoria das abordagens defende uma nítida posição valorativa: a abordagem profunda é superior à abordagem superficial, em termos de aprendizagem e desenvolvimento. Esse pensamento decorre do postulado piagetiano de que o desenvolvimento se dá principalmente por meio da interação sujeito-objeto. Quanto mais profunda a interação, mais provocada e provavelmente alterada será a estrutura cognitiva do sujeito, gerando conseqüentemente um maior desenvolvimento da capacidade de pensar e aprender. $\mathrm{Na}$ medida em que a $A P$ imprime uma interação mais forte com o objeto de conhecimento, a teoria das abordagens pressupõe que há um maior desenvolvimento cognitivo nas pessoas que possuem uma $A P$ mais desenvolvida. Portanto, a teoria das abordagens postula que a utilização da $A P$ possibilita um aprendizado escolar de melhor qualidade.

Ao salientar o papel da $A P$ junto ao desenvolvimento cognitivo dos estudantes, a teoria das abordagens defen- de que a escola tem um papel fundamental na regulação da forma como o aluno interage com os objetos formais de conhecimento. A escola tem o potencial educativo de mobilizar a expressão de zonas proximais do estudante, impulsionando-o a funcionar em nível ótimo (Fischer, Knight, \& van Parys, 1993; Kozulin, Gindis, Ageyev, \& Miller, 2003; Vygotsky, 1978). Por outro lado, a escola pode mobilizar as práticas pedagógicas de modo a enfatizar o nível funcional do aluno. Caso utilize e valorize tarefas e atividades que demandem a utilização da abordagem profunda, a escola poderá, segundo a teoria das abordagens, alterar a longo prazo o curso de desenvolvimento cognitivo e a capacidade de aprendizagem de seus alunos.

\section{Conclusão}

Os resultados do estudo podem ser sintetizados da seguinte maneira. Do ponto de vista de sua contribuição à avaliação psicológica: (a) a EABAP é um instrumento equilibrado de itens que mensura o modelo das abordagens superficial e profunda. (b) Por possuir uma escala geral e duas escalas específicas, a EABAP possibilita tanto a análise das abordagens superficial e profunda em termos de polaridades distintas (via a escala geral) quanto possibilita a análise das abordagens em separado (via as escalas específicas). (c) A EABAP traz evidências que corroboram a robustez do modelo das abordagens superficial-profunda, como sugerem Wong et al. (1996). (d) As análises das escalas específicas trazem informações relevantes a respeito de possíveis trajetórias de desenvolvimento das abordagens de aprendizagem, em função da idade e série. (e) Através das medidas da EABAP é possível às escolas planejar ações pedagógicas direcionadas à aprendizagem dos estudantes.

Do ponto de vista das limitações do estudo, (a) há a necessidade de evidências longitudinais a respeito das trajetórias de desenvolvimento das abordagens superficial e profunda. O estudo apresenta exclusivamente dados transversais, de modo que os resultados apresentados indicam exclusivamente trajetórias hipotéticas. (b) Para que a EABAP possa ser uma ferramenta efetiva no diagnóstico de estudantes predominantemente superficiais ou profundos em sua abordagem de aprendizagem, novos estudos deverão identificar os pontos de corte adequados na escala geral e nas escalas específicas. (c) Novos estudos deverão também indicar o que significa uma pessoa se localizar no ponto intermediário da escala geral. Um dos objetivos desses novos estudos envolve a investigação a respeito da relação entre a posição das pessoas na escala geral e a presença do uso estratégico das abordagens superficial e profunda para fins específicos de alto desempenho. (d) O presente estudo possui uma amostra localizada de estudantes do ensino fundamental e ensino médio de uma escola particular. Novos estudos são necessários em alunos de escolas distintas, com diferentes perfis pedagógicos e socioeconômicos. 


\section{Referências}

Almeida, L. S. (1992). Inteligência e aprendizagem: Dos seus relacionamentos à sua promoção. Psicologia: Teoria e Pesquisa, 8(3), 277-292.

Almeida, L. S. (1996). Cognição e aprendizagem: Como a sua aproximação conceptual pode favorecer o desempenho cognitivo e a realização escolar. Psicologia: Teoria, Investigação e Prática, 1, 17-32.

Beauducel, A. (2001). Problems with parallel analysis in data sets with oblique simple structure. Methods of Psychological Research Online, 6(2), 141-157.

Biggs, J. B. (1978). Individual and group differences in study processes. British Journal of Educational Psychology, 48, 266-279.

Biggs, J. B. (1985). The role of meta-learning in study processes. British Journal of Educational Psychology, 55, 185-212.

Biggs, J. B. (1987a). The Learning Process Questionnaire (LPQ): Users' manual. Hawthorn, Australia: Australian Council for Educational Research.

Biggs, J. B. (1987b). The Study Process Questionnaire (SPQ): Manual. Hawthorn, Australia: Australian Council for Educational Research.

Biggs, J. B., Kember, D., \& Leung, D. Y. P. (2001). The revised two-factor Study Process Questionnaire: R-SPQ-2F. British Journal of Educational Psychology, 71, 133-149.

Blunch, N. J. (2008). Introduction to structural equation modelling using SPSS and AMOS. Thousand Oaks, CA: Sage.

Boruchovitch, E. (1993). A Psicologia Cognitiva e a metacognição: Novas perspectivas para o fracasso escolar brasileiro. Tecnologia Educacional, 22(110/111), 22-28.

Boruchovitch, E. (1999). Estratégias de aprendizagem e desempenho escolar: Considerações para a prática educacional. Psicologia: Reflexão e Crítica, 12(2), 361-373.

Byrne, B. (2001). Structural equation modeling with AMOS: Basic concepts, applications and programming. Mahwah, NJ: Lawrence Erlbaum.

Da Silva, A. L., \& Sá, I. (1993). Saber estudar e estudar para saber. Ciências da Educação. Porto, Portugal: Porto.

Dawson-Tunik, T. L., Commons, M., Wilson, M., \& Fischer, K. W. (2005). The shape of development. European Journal of Developmental Psychology, 2, 163-195.

De Corte, E. (2000). Marrying theory building and the improvement of school practice: A permanent challenge for instructional psychology. Learning and Instruction, 10(3), 249-266.

Dembo, M. H. (1994). Applying educational psychology. New York: Longman.

Duarte, A. (2002). Aprendizagem, ensino e aconselhamento educacional: Uma perspectiva cognitivo-motivacional. Porto, Portugal: Porto.

Entwistle, N. J. (1988). Motivational factors in students' approaches to learning. In R. R. Schmeck (Ed.), Learning strategies and learning styles (pp. 21-51). New York: Plenum Press.

Entwistle, N. J., McCune, V., \& Walker, P. (2001). Conceptions, styles and approaches within higher education: Analytic abstractions and everyday experience. In R. Stenberg \& L-F. Zang (Eds.), Perspectives on cognitive, learning and thinking styles (pp. 211-245). Mahwah, NJ: Lawrence Erlbaum.

Entwistle, N. J., Meyer, J. H. F., \& Tait, H. (1991). Students failure: Disintegrated perceptions of studying and the learning environment. Higher Education 21, 249-261.
Entwistle, N. J., \& Ramsden, P. (1983). Understanding student learning. London: Croom Helm.

Fischer, K. W., \& Bidell, T. R. (2006). Dynamic development of action, thought, and emotion. In W. Damon \& R. M. Lerner (Eds.), Handbook of Child Psychology: Vol. 1. Theoretical models of human development. (pp. 313-399). New York: Wiley.

Fischer, K. W., \& Kennedy, B. (1997). Tools for analyzing the many shapes of development: The case of self-in-relationships in Korea. In E. Amsel \& K. A. Renninger (Eds.), Change and development: Issues of theory, method, and application (pp. 117-152). Mahwah, NJ: Lawrence Erlbaum.

Fischer, K. W., Knight, C., \& van Parys, M. (1993). Analyzing diversity in developmental pathways: Methods and concepts. In R. Case \& W. Edelstein (Eds.), The new structuralism in cognitive development: Theory and research on individual pathways. Contributions to human development (pp. 33-56). New York: Karger.

Frazier, T. W., \& Youngstrom, E. A. (2007). Historical increase in the number of factors measured by commercial tests of cognitive ability: Are we overfactoring? Intelligence, 35, 169-182.

Garner, R., Hare, V. C., Alexander, P., Haynes, J., \& Winograd, P. (1984). Inducing use of a text lookback strategy among unsucessful readers. American Educational Research Journal, 21, 789-798.

Gomes, C. F. (2005). Escala de avaliação de processos de estudo (EAPE). In Psicologia.com.pt - O Portal dos Psicólogos. Retrieved September 22, 2008, from http:// www.psicologia.com.pt

Guadagnoli, E., \& Velicer, W. F. (1988). Relation of sample size to the stability of component patterns. Psychological Bulletin, 103(2), 265-275.

Hattie, J., Biggs, J., \& Purdue, N. (1996). Effects of learning skills interventions on students: A meta analysis. Review of Educational Research, 66(2), 99-136.

Henson, R. K., \& Roberts, J. K. (2006). Use of exploratory factor analysis in published research: Common errors and some comment on improved practice. Educational and Psychological Measurement, 66(3), 393-416.

Holt, J. (1982). How children fail. New York: Delta.

Kozulin, A., Gindis, B., Ageyev, V., \& Miller, S. M. (2003). Vygotsky's educational theory in cultural context. Cambridge, UK: Cambridge University Press.

Marton, F. (1975). What does it take to learn? Some implications of an alternative view of learning. In N. Entwistle \& D. Hounsell (Eds.), How students learn (pp. 125-138). Lancaster, UK: University of Lancaster.

Marton, F., \& Säljö, R. (1976a). On qualitative differences in learning: I - Outcome and Process. British Journal of Educational Psychology, 46, 4-11.

Marton, F., \& Säljö, R. (1976b). On qualitative differences in learning: II. Outcome as a function of the learner's conception of the task. British Journal of Educational Psychology, 46, 115-127.

Mettrau, M. B., \& Mathias, M. T. (1998). O papel social da prática pedagógica do professor na promoção das capacidades sócio-cognitivo-afetivas do alunado. Tecnologia Educacional, 26(141), 30-34.

Nisbet, J., \& Schucksmith, J. (1986). Learning strategies. London: Routledge \& Kegan Paul.

Pozo, J. I. (1996). Estratégias de aprendizagem. In C. Coll, J. Palácios, \& A. Marchesi (Eds.), Desenvolvimento psicológico e educação: Psicologia da Educação (pp. 176-197). Porto Alegre, RS: Artes Médicas. 
Gomes, C. M. A., Golino, H. F., Pinheiro, C. A. R., Miranda, G. R. \& Soares, J. M. T. (2011). Validação da Escala de Abordagens de Aprendizagem (EABAP) em uma Amostra Brasileira.

Pressley, M., \& Levin, J. R. (1983). Cognitive strategy research: Psychological Foundations. New York: Springer-Verlag.

Riding, R., \& Rayner, S. (Eds.). (1998). Cognitive styles and learning strategies: Understanding style differences in learning and behaviour. London: David Fulton.

Rosário, P., Almeida, L., Núñez, J. C., \& González-Pienda, J. A. (2004). Abordagem dos alunos à aprendizagem: Análise do construto. Psico-USF, 9(2), 117-127.

Struyven, K., Dochy, F., Janssens, S., \& Gielen, S. (2006). On the dynamics of students' approaches to learning: The effects of the teaching/learning environment. Learning and Instruction, 20, 1-16.

Vermunt, J., \& Vermetten, Y. (2004). Patterns in student learning: Relationships between learning strategies, conceptions of learning and learning orientations. Educational Psychology Review, 16, 359-384.

Vygotsky, L. S. (1978). Mind in society: The development of higher psychological processes. Cambridge, MA: Harvard University Press.

Weinstein, C. E., \& Mayer, R. E. (1985). The teaching of learning strategies. In M. Wittrock (Ed.), Handbook of research on teaching (pp. 315-327). New York: Macmillan.

Wild, M., \& Quinn, C.N. (1998). Implications of educational theory for the design of instructional multimedia. British Journal of Educational Technology, 29, 73-82.

Wong, N. Y., Lin, W. Y., \& Watkins, D. (1996). Cross-cultural validation of models of approaches to learning: An application of confirmatory factor analysis. Educational Psychology, 16(3), 317-327.

Yan, Z., \& Fischer, K. W. (2002). Always under construction: Dynamic variations in adult cognitive development. Human Development, 45, 141-160. 